\title{
Experimental investigation of left-right asymmetry in photon-atom interaction
}

\author{
S. Ricz, ${ }^{1,2}$ T. Buhr, ${ }^{3}$ Á. Kövér, ${ }^{1}$ K. Holste, ${ }^{2}$ A. Borovik, Jr., ${ }^{2}$ S. Schippers, ${ }^{2}$ D. Varga, ${ }^{1}$ and A. Müller ${ }^{2}$ \\ ${ }^{1}$ Institute for Nuclear Research, Hungarian Academy of Sciences (Atomki), P.O. Box 51, H-4001 Debrecen, Hungary \\ ${ }^{2}$ Institute for Atomic and Molecular Physics, Justus-Liebig University Giessen, D-35392 Giessen, Germany \\ ${ }^{3}$ Physikalisch-Technische Bundesanstalt, Bundesallee 100, D-38116 Braunschweig, Germany
}

(Received 8 April 2014; published 10 July 2014)

\begin{abstract}
Single ionization of noble-gas atoms by linearly polarized synchrotron radiation has been studied by employing angle- and energy-resolved photoelectron spectroscopy. The measurements were carried out in the plane defined by the momentum and polarization vectors of the photon. Parameters describing the left-right asymmetry (LRA; relative to the photon propagation direction) of the photoelectron angular distribution were determined experimentally for the $s$ shells of $\mathrm{He}, \mathrm{Ne}, \mathrm{Ar}, \mathrm{Kr}$, and $\mathrm{Xe}$ atoms and $\mathrm{H}_{2}$ molecules and for the $p$ shells of $\mathrm{Ne}, \mathrm{Ar}$, $\mathrm{Kr}$, and $\mathrm{Xe}$ atoms. The values of the left-right asymmetry differ significantly from zero for both subshells. The photon and photoelectron energy dependence of the LRA parameters is presented also. Possible experimental and instrumental sources that could generate asymmetry are discussed and excluded as well.
\end{abstract}

DOI: 10.1103/PhysRevA.90.013410

PACS number(s): $32.80 . \mathrm{Fb}, 32.80 . \mathrm{Hd}$

\section{INTRODUCTION}

According to quantum mechanics, electromagnetic atomic transitions have space inversion symmetry. The electromagnetic interactions among the atomic electrons and nucleus as well as between the ionized and excited particles are assumed to conserve parity. Consequently, for photoionization with linearly polarized light the angular distributions of the emitted particles or quanta should show left-right symmetry relative to the photon propagation direction. In other words, the right-side intensity of ejected particles with respect to the beam direction is equal to the left-side intensity.

The angular distribution of photoelectrons can be expressed by the following formulas for unpolarized [Eq. (1)] and entirely (100\%) linearly polarized [Eq. (2)] light including first-order nondipole correction $[1,2]$ :

$$
\frac{d \sigma_{n l}}{d \Omega}=\frac{\sigma_{n l}}{4 \pi}\left[1-\frac{1}{2} \beta P_{2}(\cos \psi)+\left(\delta+\frac{1}{2} \gamma \sin ^{2} \psi\right) \cos \psi\right],
$$

$$
\begin{aligned}
\frac{d \sigma_{n l}}{d \Omega}= & \frac{\sigma_{n l}}{4 \pi}\left[1+\frac{1}{2} \beta\left(3 \sin ^{2} \psi \cos ^{2} \chi-1\right)\right. \\
& \left.+\left(\delta+\gamma \sin ^{2} \psi \cos ^{2} \chi\right) \cos \psi\right]
\end{aligned}
$$

where $\beta$ is the dipole $(E 1)$ and $\delta$ and $\gamma$ are the nondipole $(M 1, E 2)$ anisotropy parameters. The definition of the angles is shown in Fig. 1(a). The polar $(\psi)$ and the azimuthal $(\chi)$ angles of the photoelectrons are measured with respect to the momentum $(\mathbf{k})$ and polarization $(\mathbf{P})$ vectors of the photons, respectively [Fig. 1(a)]. $P_{2}$ is the second-order Legendre polynomial, and $\sigma_{n l}$ is the photoionization cross section for the $n l$ shell. An example of the photoelectron angular distribution is shown in Fig. 1(b). As Fig. 1 shows, the nondipole correction produces only a forward-backward asymmetry relative to the photon momentum vector in the case of linearly polarized and unpolarized photon beams. Therefore, it does not break the symmetry around the propagation direction of the photon. For a linearly polarized photon beam a right-handed $X Y Z$ coordinate system [Fig. 1(a)] can be defined in the following way [3]: The photon momentum vector $\vec{k}$ points to the direction of the $X$ axis and the photon polarization vector $\vec{P}$ is aligned along the $Z$ axis. The $Y$ axis is perpendicular to the $X Z$ plane and points upward. [The $\vec{E}(r, t)$ electric vector of the incoming photon oscillates in the $X Z$ plane.] Positive $Z$ defines the right-hand side (R), and negative $Z$ defines the left-hand side (L) relative to the propagation direction of the incoming light. The mirror plane [ $X Y$ plane in Fig. 1(a)] is perpendicular to the polarization direction of the photon beam. Using the above described coordinate system the left-right asymmetry parameter $A_{L R}$ for photoelectron emission may be defined as [3]

$$
A_{L R}=\frac{\sigma_{L}-\sigma_{R}}{\sigma_{L}+\sigma_{R}}
$$

where $\sigma_{L}$ and $\sigma_{R}$ are the cross sections for photoelectron emission to the left side ( $\psi$ varies between $0^{\circ}$ and $180^{\circ}$, clockwise) and to the right side ( $\psi$ varies between $0^{\circ}$ and $-180^{\circ}$, counterclockwise) relative to the photon propagation direction, respectively.

In our previous paper [3] left-right asymmetry (LRA) has been observed in the outer $s$-shell double-differential photoionization cross sections for linearly polarized synchrotron radiation. The investigated angular ranges for photoelectron emission were $\chi= \pm 1.7^{\circ}$ and $\psi=0^{\circ} \pm 180^{\circ}$. For the interpretation of the experimental data two possible explanations for the observed LRA were suggested and excluded: (a) The LRA is the result of the weak interaction among the nucleons and the atomic electrons mediated by the exchange of $Z_{0}$ bosons. However, the experimental atomic mass dependence and the order of magnitude of the experimental values strongly differ from the theoretical predictions published in [3]. This indicates that the observed left-right asymmetry cannot be interpreted as a result of the weak interaction. (b) The LRA is associated with the interactions of ultrashort laser pulses [4]. When the photon wave packet is extremely short and the phase difference of the carrier envelope is constant, LRA (or virtual parity violation) may exist. However, such a time structure of 

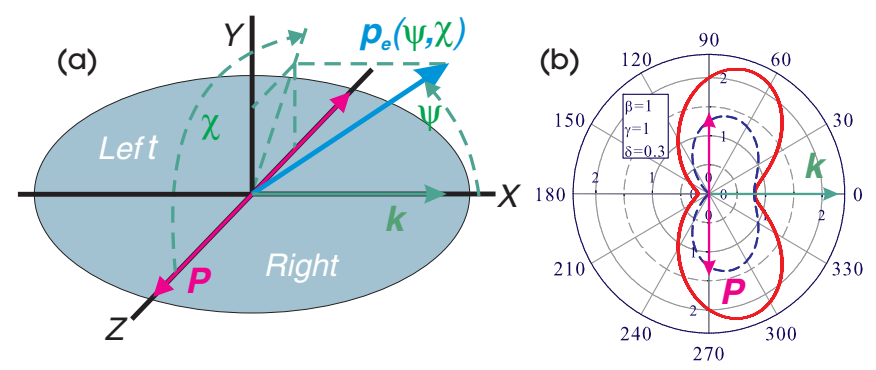

FIG. 1. (Color online) (a) Definition of the coordinate system. (b) An example of the double-differential cross section of photoelectrons in the scattering $(\mathbf{P}, \mathbf{k})$ plane. The solid and the dashed lines represent the angular distribution of emitted photoelectrons for linearly polarized and unpolarized light calculated from Eqs. (1) and (2), respectively, for the given parameter values.

photon wave packets emitted by a synchrotron light source has not been observed so far.

The aim of the present work is to check the previously observed left-right asymmetry [3] by applying different experimental setups (electron spectrometer and synchrotron). The measurements were carried out at the DORIS III synchrotron light source [5-8], while the previous experiments [3] were done at the MAX II synchrotron $[9,10]$. The preliminary results were published by Ricsóka et al. [11]. Furthermore, our experimental investigation has been extended to the $p$ shells of $\mathrm{Ne}, \mathrm{Ar}, \mathrm{Kr}$, and $\mathrm{Xe}$ using nearly the same photon energies as for the corresponding $s$ shells. Moreover, the previous spectra measured at the MAX II synchrotron were also reevaluated for the determination of the LRA parameters. In the Appendix the possible experimental and instrumental effects which may cause LRA and their magnitudes are carefully analyzed.

\section{EXPERIMENT AND DATA EVALUATION}

Two different synchrotrons were used in the measurements: the DORIS III storage ring at HASYLAB, Hamburg, Germany (beam line BW3) and the MAX II at MAX-lab, Lund, Sweden (beam line I411). Positrons are used to produce synchrotron radiation in DORIS III, and the operating energy is $4.45 \mathrm{GeV}$. This energy is almost three times higher than that of MAX II $(1.5 \mathrm{GeV})$ where electrons are accelerated. The photon source at beam line BW3 was composed of two undulators with overlapping energy ranges, while at beam line I411 of the MAX II synchrotron one undulator is used. Both beam lines were equipped with SX-700 monochromators.

The ejected photoelectrons were analyzed with the ESA22G and ESA-22L [12] electrostatic electron spectrometers. ESA-22G is a slightly modified version of the ESA-22L electron spectrometer developed at Atomki, Debrecen, Hungary. The main working principles and geometric dimensions are the same for both spectrometers. A sketch of the analyzer can be seen in Fig. 2. It consists of spherical and cylindrical electrostatic mirrors. The spherical part focuses the electrons from the scattering plane ( $X Z$ plane) to the entrance slit of the cylindrical mirror analyzer, which performs the energy analysis of the electrons.

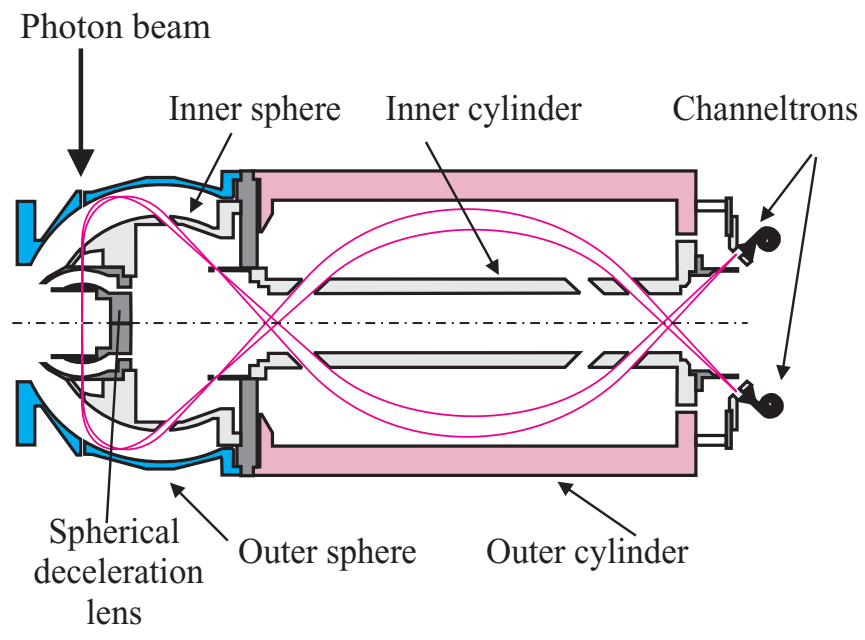

FIG. 2. (Color online) Schematic cross section of an ESA-22type electron spectrometer.

A spherical deceleration lens is placed around the source volume to improve the energy resolution of the equipment. Due to the radial electrostatic field of the decelerator lens and of the whole spectrometer the polar angles of the emitted electrons ( $\psi$ in Fig. 1) are conserved from the target all the way to the detectors. The analyzer and the interaction region are shielded from the Earth's magnetic field by three layers of $\mu$-metal sheets. The residual magnetic field in the scattering plane and in the analyzer is less than $500 \mathrm{nT}$ for both electron spectrometers.

The photoelectrons were detected at $\chi=0^{\circ}$ azimuthal and at 22 polar angles $(\psi)$ in $15^{\circ}$ steps between $0^{\circ}$ and $\pm 180^{\circ}$ relative to the photon momentum vector (Fig. 1). The acceptance angles of each channeltron were $\Delta \psi= \pm 5^{\circ}$ and $\Delta \chi= \pm 1.7^{\circ}$. An important difference between the two analyzers is the availability of two additional observation angles in $\mathrm{ESA}-22 \mathrm{G}$ (at $90^{\circ}$ and $-90^{\circ}$ with respect to the photon momentum vector) in the direction of the oscillating electric field vector. Another difference between the two spectrometers is the modified geometry of the gas target. (The gas nozzle is located in the symmetry axes of both analyzers.) In the case of ESA-22G a simple tube is used as a gas nozzle, and the gas flows upwards; in ESA-22L [12] a channel plate is fixed to the end of the nozzle, and the gas is directed downwards. In the latter case a more directed gas flow is expected. In the DORIS III experiment a new analyzer control and a faster signal-processing system as well as new software were used to control the spectrometer and to collect data.

In the recent experiments at DORIS III the LRA parameters were determined at 203.3-eV photoelectron energy in the $X Z$ plane (it is the same plane as in the previous experiment [3]). The source size was $\pm 0.85 \mathrm{~mm}$, determined by the geometry of ESA-22G in the direction perpendicular to the $X Z$ plane. The photon energies were chosen such that the kinetic energies of the photoelectrons, ejected from the outer $s$ shells, were nearly the same as those of the Ar LMM Auger electrons. (The corresponding photon energy range was 226.7-256.3 eV.) In this way identical experimental conditions were ensured for the detection of Auger electrons and photoelectrons. The 


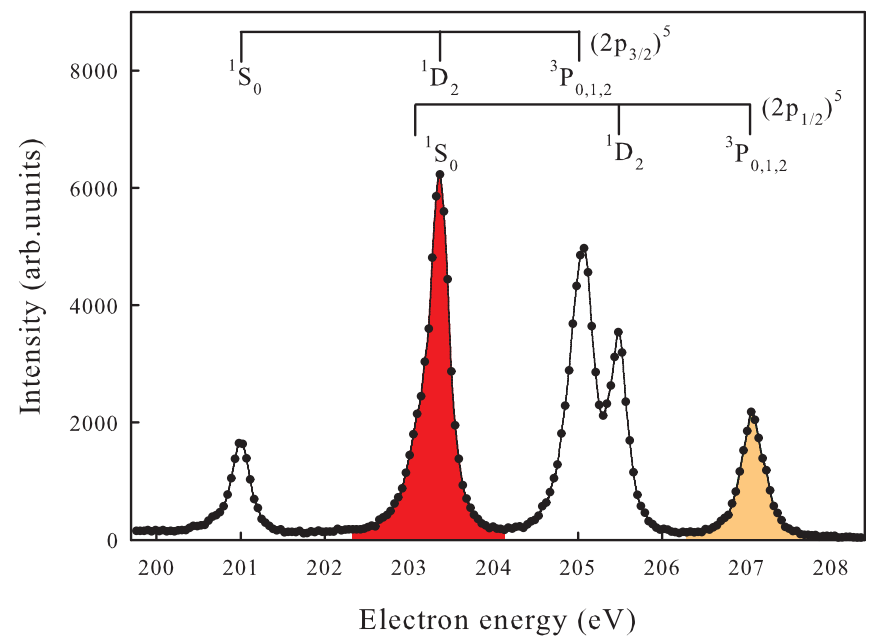

FIG. 3. (Color online) The Ar $L_{2,3}-M_{2,3} M_{2,3}$ Auger group measured at 461.2-eV photon energy with a 500- $\mu$ m monochromator slit size. The colored Auger peaks were used to normalize the photoelectron spectra. The dark red (dark gray) shaded peak $(\approx 203.3 \mathrm{eV})$ was employed in the DORIS III experiment, while in the MAX II experiment [3] the light orange (light gray) line $(\approx 207.0 \mathrm{eV})$ was used.

energy and angular distributions of the Auger electrons and photoelectrons were measured at $80-\mathrm{eV}$ pass energy (and the energy resolution of the analyzer was about $160 \mathrm{meV}$ FWHM). For $\mathrm{He} 1 s$ and $\mathrm{Ne} 2 s$ shells a 500- $\mu \mathrm{m}$-wide monochromator slit size (corresponding to a bandwidth of $40 \mathrm{meV}$ ) and for $\mathrm{Ar}$ $3 s$ and Xe $5 s$ shells a slit size of $180 \mu \mathrm{m}$ (corresponding to a bandwidth of $13 \mathrm{meV}$ ) were used. This allowed us (together with the high-energy resolution of the spectrometer) to separate the satellite photolines from the diagram lines.

We have not found any indication in the literature for nonzero asymmetry parameters in the case of the Auger process for atoms. Therefore, the intensities of all photoelectron spectra measured at different angular channels were normalized to the intensity of the Ar LMM Auger peaks. This normalization was necessary since the individual detection efficiencies of the channeltrons were not known. In the previous experiments [3] the isotropic but weak $\operatorname{Ar} L_{2}-M_{2,3} M_{2,3}$ ${ }^{3} P_{0,1,2}$ Auger transitions (at $207 \mathrm{eV}$, light orange peak in Fig. 3) were used to normalize the photoelectron spectra. In this measurement the almost isotropic $\operatorname{Ar} L_{3}-M_{2,3} M_{2,3}$ ${ }^{1} D_{2}$ diagram Auger line at 203.3-eV kinetic energy was used for normalization [see Fig. 3, dark red (dark gray) peak]. It was measured at $461.2 \mathrm{eV}$ incident photons using a $500-\mu \mathrm{m}$ monochromator slit size. This line was chosen because its intensity is nearly three times higher than that of the isotropic Ar $L_{2}-M_{2,3} M_{2,3}{ }^{3} P_{0,1,2}$ Auger transition. Its anisotropy is weak according to theoretical calculations.

The angular distribution of Auger electrons resulting from the decay of a $n p_{3 / 2}$ vacancy (with principal quantum number $n=2,3,4, \ldots)$ is given as $[13,14]$

$$
\frac{d \sigma(\theta)}{d \Omega}=\frac{\sigma_{0}}{4 \pi}\left[1+\beta_{2} P_{2}(\cos \theta)\right]
$$

where $\frac{d \sigma(\theta)}{d \Omega}$ is the double-differential cross section of the Auger electron production at angle $\theta$ with respect to the photon beam direction, $\Omega$ is the solid angle, $\sigma_{0}$ is the total cross section, $\beta_{2}$ is the anisotropy parameter, and $P_{2}(\cos \theta)$ is the second-order Legendre polynomial. $\beta_{2}$ is related to the alignment parameter $\mathscr{A}_{2}$ in the following way:

$$
\beta_{2}=\alpha_{2} \mathscr{A}_{2},
$$

where $\alpha_{2}$ is the anisotropy coefficient. The alignment parameter depends only on the ionization process, while the anisotropy coefficient depends only on the decay process (two-step model). In the case of the $\operatorname{Ar} L_{2}-M_{2,3} M_{2,3}{ }^{3} P_{0,1,2}$ transitions there is no alignment because the $J_{i}$ total angular momentum of the initial state equals $1 / 2\left(\mathscr{A}_{2}=0\right)[13,15]$. This means that the angular distributions of these Auger electrons are isotropic. The anisotropy parameter $\beta_{2}$ of the $L_{3}-M_{2,3} M_{2,3}{ }^{1} D_{2}$ transition calculated with Eq. (5) is approximately -0.0288 (using the data in Refs. [14,16] for $\mathscr{A}_{2}$ and in Ref. [17] for $\alpha_{2}$ ). Due to this small value the above-mentioned Auger transition can be used for normalization. Since the angular distribution of the Auger electrons is symmetric with respect to the photon beam momentum vector [as Eq. (4) shows], the normalization of the photoelectron angular distribution to an anisotropic angular distribution of Auger electrons cannot introduce any LRA.

The linear polarization of the photon beam was monitored by recording the angular distribution of $\mathrm{Ne} 2 s$ photoelectrons at $250-\mathrm{eV}$ photon energy where the nondipole contribution is negligible [18]. The radiation was found to be completely linearly polarized: $100 \%$ within $2 \%$ uncertainty.

The measurement and the evaluation of the experimental data were performed in a manner similar to the one described in Ref. [3]. The photon flux was measured by a photodiode. The collection times of the photoelectrons were several tens of seconds at each energy point, and the energy sweeps were repeated 10 to 90 times depending on the magnitude of the photoionization cross sections. Before and after the collection of photoelectron spectra an Ar LMM Auger spectrum was recorded. After linear background substraction the angular distribution of the photoelectrons was determined by normalizing the intensity of the photoelectron line at each angle to the area of the selected (nearly isotropic) Auger peak in every single angular channel. The relative double-differential cross sections obtained at different emission angles were summed for the left and right spectrometers halves, separately. Finally, the asymmetry parameter was calculated by using Eq. (3), and its error was estimated from the statistical uncertainty, background substraction, normalization, and reproducibility.

Possible experimental or instrumental sources that could generate left-right asymmetry are discussed and their magnitudes are estimated in the Appendix.

\section{RESULTS AND DISCUSSION}

Figure 4 compares the experimental values of the LRA parameters $A_{L R}$ determined for the $s$-shell photoelectrons for the $\mathrm{H}_{2}$ molecule and noble-gas atoms as a function of the atomic mass. Figure 4 shows four measured data sets. Three of them were collected at the MAX II synchrotron and published earlier [3]. One of them was measured when the spectrometer system was rotated by $180^{\circ}$ horizontally around the analyzer axis (inverted triangles). The fourth data set (open 


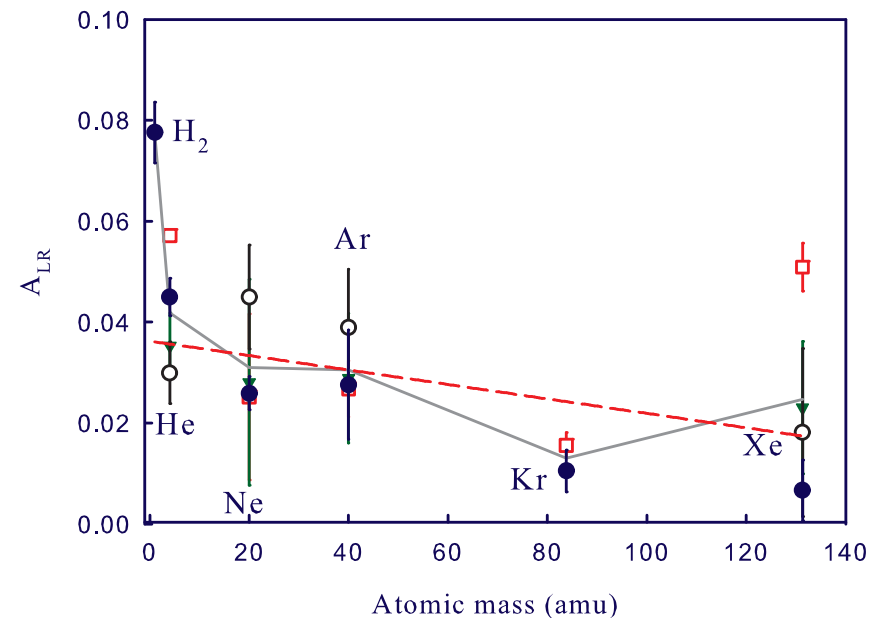

FIG. 4. (Color online) Comparison of the experimental LRA parameters $A_{L R}$ for $\mathrm{H}_{2}$ molecule and outer $s$ shells of noble gases. Three data sets were collected at MAX II [3], and one was measured at DORIS III [11]: solid circles, first observation of the nonzero asymmetry parameters at MAX II; squares, results of the repeated experiment after the careful test of the experimental system at MAX II; inverted triangles, asymmetry parameters after rotation of the spectrometer system by $180^{\circ}$ horizontally at MAX II; open circles, data measured at DORIS III. The solid line shows the average LRA parameters obtained from the results of different measurements. The dashed line is the linear fit for the mean values of the noble gases.

circles) was measured at the DORIS III storage ring with the ESA-22G electron spectrometer system [11]. All data sets show nearly the same asymmetry. It is hard to believe that the time structure and the phase differences between the carrier envelopes of the photons are the same for both synchrotrons. This indicates that the asymmetric photoelectron emission is not an instrumental or experimental effect and originates from the photoionization process itself. All four data sets show definite positive asymmetry parameters for photoionization of the $s$ shells of the studied targets.

The LRA was also investigated for the Ne $2 p, \operatorname{Ar} 3 p, \mathrm{Kr}$ $4 p$, and $\mathrm{Xe} 5 p$ shells at the MAX II synchrotron using the same experimental conditions as for the $s$ shells. Figure 5 shows the asymmetry parameters as a function of the atomic mass (Ar $2 p$ asymmetry parameters were added to the figure from [3,19]). As Fig. 5 shows, nearly constant negative values were determined for the asymmetry parameters of $\mathrm{Ne}, \mathrm{Kr}$, and $\mathrm{Xe}$ atoms. Comparing the results to the data measured for $s$ shells, there are significant differences. The sign of the values are opposite. Considering only the atomic targets, the absolute values of LRA are higher for the $s$ shells than for the $p$ shells. [The mean asymmetry parameter is $A_{L R}^{s}=0.028(5)$ for the $s$ shell without $\mathrm{H}_{2}$ and is $A_{L R}^{p}=-0.014(1)$ for the $p$ shell without Ar. The standard errors are in the brackets.] The LRA data for $s$ shells are decreasing slightly with increasing atomic mass (see dashed line in Fig. 4), and the slope and intercept of the linear fit are $-1.4(7) \times 10^{-4}$ and $0.036(5)$, respectively. (The $\mathrm{H}_{2}$ data were not taken into account in the fit.) The experimental values are nearly constant for the $p$ shells (except for Ar). These differences (see Figs. 4 and 5) indicate that the unknown correlation between the photon and

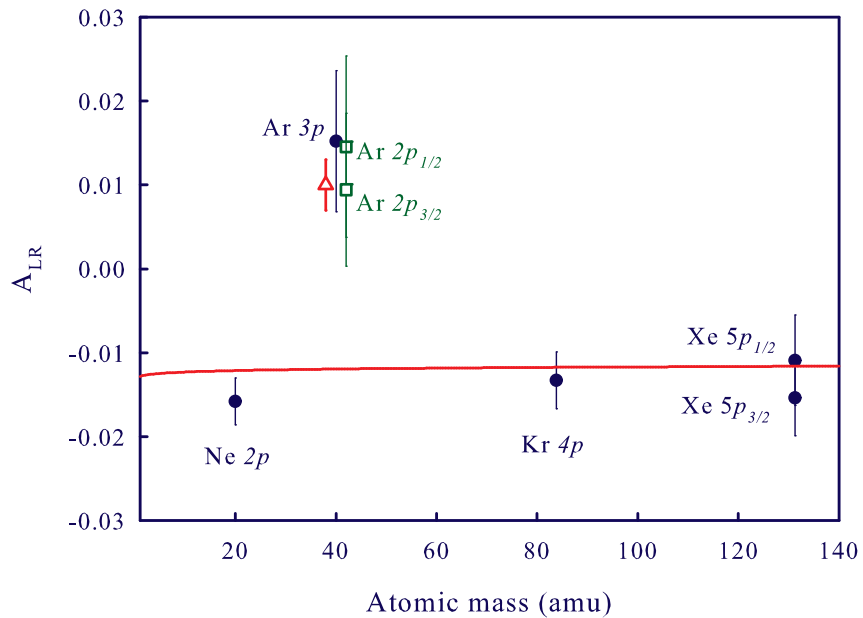

FIG. 5. (Color online) The experimental values of the asymmetry parameters $A_{L R}$ for the $p$ shells of $\mathrm{Ne}, \mathrm{Ar}, \mathrm{Kr}$, and $\mathrm{Xe}$ atoms. Circles, present work; squares, previously measured data for $\operatorname{Ar} 2 p$ shells [3]; triangles, Ar $2 p$ data measured by Heinasmaki et al. [19].

atom is sensitive to the angular momentum of atomic shells. This shows that the asymmetry is an intrinsic behavior of a photon-atom or photon-molecule interaction.

In contrast to the outer $p$-shell data the values of the asymmetry parameter for $\operatorname{Ar} 2 p$ and $3 p$ shells are positive (Fig. 5). For further investigation of this behavior Fig. 6 shows the dipole anisotropy and asymmetry parameters for $3 p$ photoelectrons of $\mathrm{Ar}$ as a function of the photon energy. The deviation between the measured [20] and calculated [2] anisotropy parameters $\beta$ starts at $125-\mathrm{eV}$ photon energy, which is far from the $2 p$ photoexcitation or photoionization threshold. It is an indication of the strong correlation between the $3 p$ and $2 p$ shells of Ar. This may be the reason why the LRA

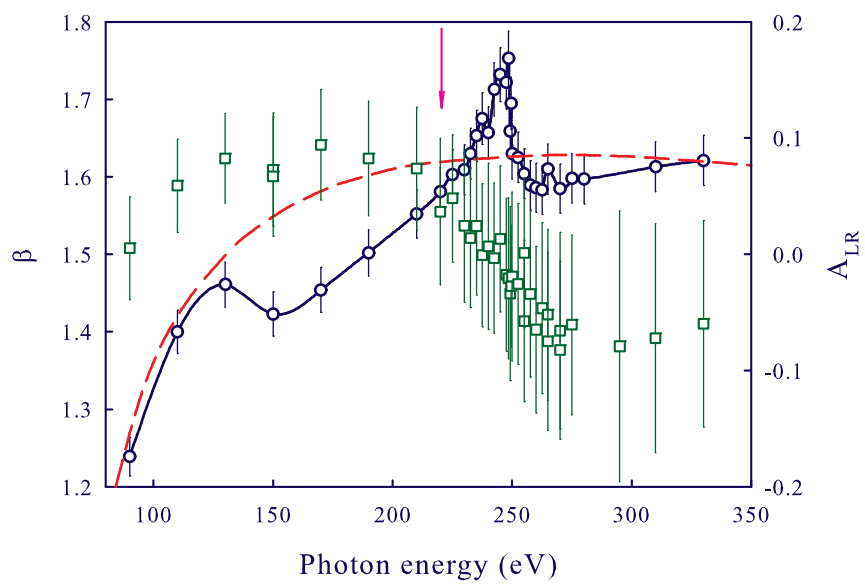

FIG. 6. (Color online) Dipole anisotropy $\beta$ and LRA parameters $A_{L R}$ for the angular distribution of $3 p$ photoelectrons of $\mathrm{Ar}$ as a function of the photon energy. Circles show experimental $\beta$ anisotropy parameters from [20]. The line is to guide the eye. Squares show experimental LRA parameters. The dashed red line shows the theoretical calculation for the anisotropy $\beta$ parameter by Derevianko and Johnson [2]. The arrow denotes the photon energy where the asymmetry parameter was presented in Fig. 5. 


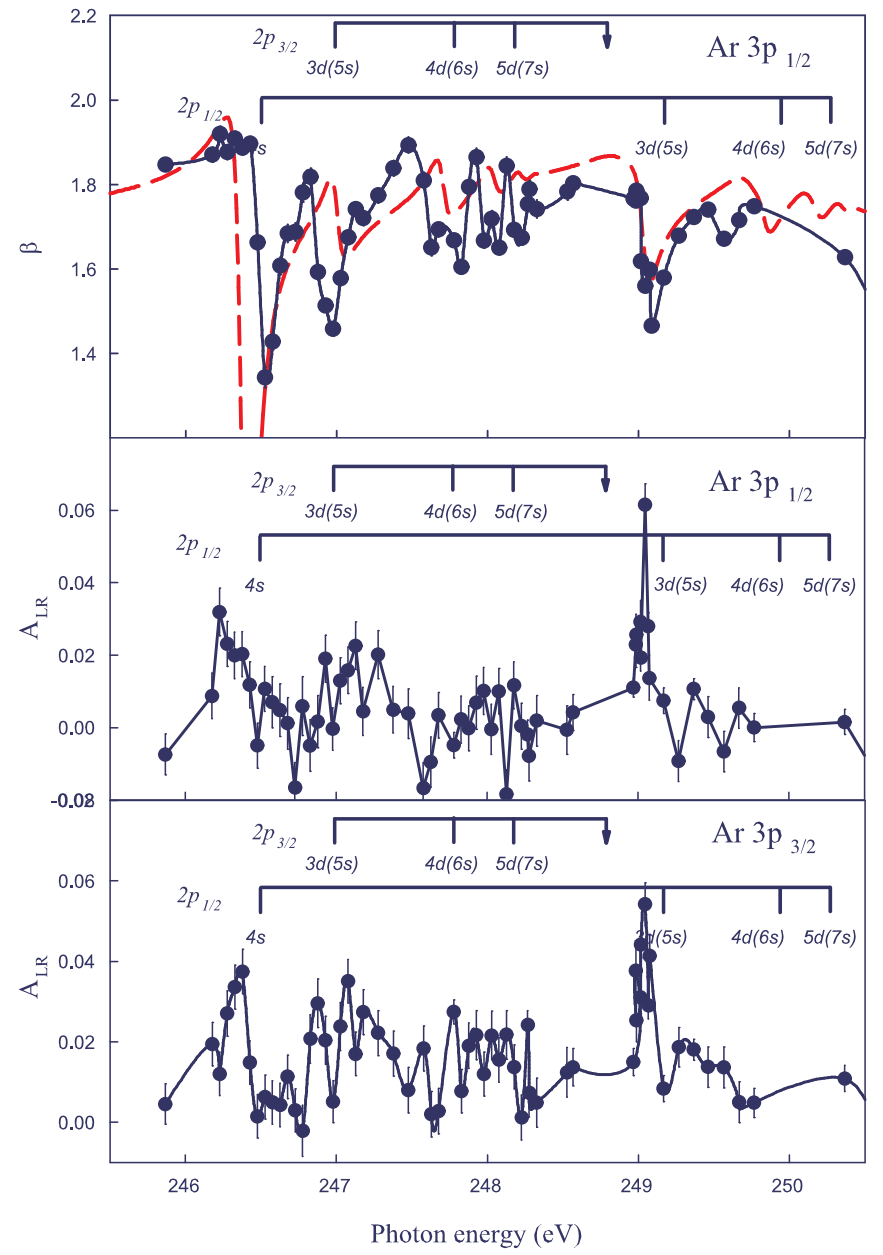

FIG. 7. (Color online) Dipole anisotropy $\beta$ (top panel for $\mathrm{Ar}$ $3 p_{1 / 2}$ ) [20] and LRA parameters $A_{L R}$ of $\mathrm{Ar} 3 p_{1 / 2}$ (middle panel) and $3 p_{3 / 2}$ shells (bottom panel) in the photon energy range where the resonant excitations of Ar $2 p$ shell exist. The vertical lines denote the positions of the resonances. The dashed line in the top panel is the theoretical calculation of Gorczyca and Robicheaux [21].

parameters for $3 p$ and $2 p$ photoelectrons are positive and roughly the same.

In our previous paper [20] a detailed investigation of the Ar $3 p$ photoelectron angular distribution was carried out. The angular anisotropy parameters for the $(2 p)^{-1}-n s / m d$ resonant photon energy region were determined with highenergy resolution and with narrow photon bandwidth. Here the LRA parameters for the $\operatorname{Ar} 3 p_{1 / 2}$ and $3 p_{3 / 2}$ photoelectron angular distributions are presented as a function of the photon energy (Fig. 7). Both the angular anisotropy $\beta$ and LRA parameters $A_{L R}$ vary strongly in the vicinity of $(2 p)^{-1}-n s / m d$ resonances. These variations are extremely sharp around the $3 d(5 s)$ resonance. We think that such sharp resonances cannot be produced by any experimental error.

Figure 8 shows the measured values for the LRA parameter as a function of the photoelectron energy for different shells of Ar and Xe. In spite of the large error bars the data are very consistent, and the spread is small, indicating that the systematic errors were probably overestimated. The values show definite structure with a zero crossing. This shape

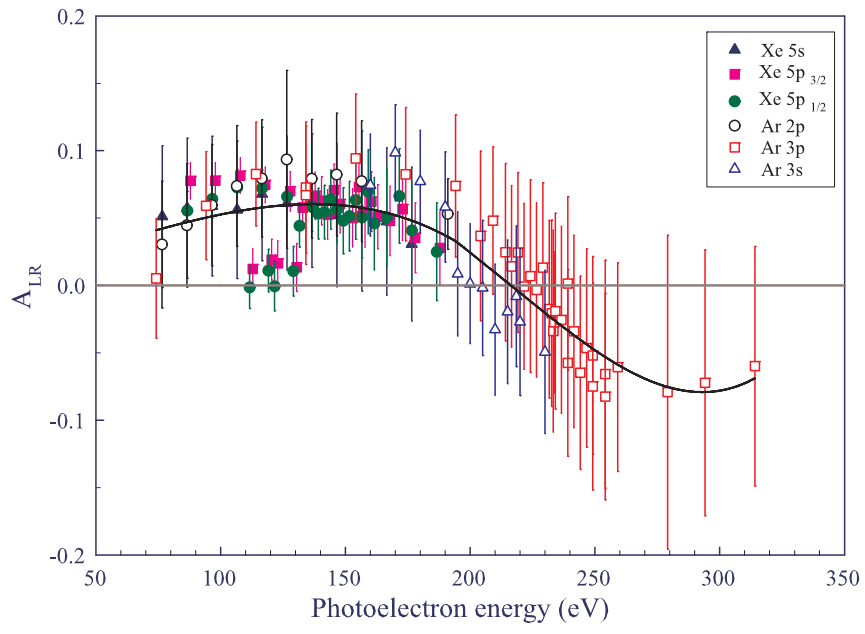

FIG. 8. (Color online) The photoelectron energy dependence of the experimental LRA parameter $A_{L R}$ in the cases of $s$ and $p$ shells for $\mathrm{Ar}$ and Xe atoms. The solid line has been obtained by smoothing the data.

suggests a general tendency for the asymmetry parameters. Few experimental values measured for $\mathrm{Xe} 5 p_{1 / 2}$ and $5 p_{3 / 2}$ are outside of the general trend. This deviation may be induced by $(4 p)^{-1}-n s / m d$ resonant excitations [22]. The photoelectron energy dependence of the LRA parameter is approximately the same for the $s$ and $p$ shells. However, Fig. 8 shows that the zero-crossing energies may be different for the $s$ and $p$-shell photoelectrons. Further experimental studies are necessary to understand the photoelectron energy dependence of the LRA parameters for different atomic shells with smaller errors. An interesting measurement would be to investigate the energy dependence of LRA with different polarizations of the photon beam (left- and right-circularly polarized and unpolarized beams).

\section{CONCLUSION}

The left-right asymmetry parameters were measured for photoionization of the outer $s$ shells of noble-gas atoms and $\mathrm{H}_{2}$ molecules using linearly polarized synchrotron radiation [3]. The aim of the present investigations was to verify the exitance of the earlier found left-right asymmetry in the angular distribution of photoelectrons. The recent measurements were carried out in a totally different experimental environment (applying another synchrotron light source and a different electron spectrometer). The measured asymmetry parameters $A_{L R}$ resulting from two different studies are in good agreement and significantly differ from zero. Both data sets show a decrease of $A_{L R}$ with increasing atomic mass for $s$ shells. The experimental LRA parameters of $\mathrm{Ne} 2 p, \operatorname{Ar} 3 p, \mathrm{Kr} 4 p$, and Xe $5 p$ were presented as well. The sign and the shape of the measured data for $p$ shells differ from the data determined for $s$ shells. This indicates that the unknown correlation is sensitive to the angular momentum of the atomic shells in photon-atom interactions.

The data measured and published earlier were evaluated again for the determination of the LRA parameters. In the case of Ar $3 p$ photoelectrons the asymmetry parameters oscillate 
strongly in the vicinity of the $(2 p)^{-1}-n s / m d$ resonances. The photoelectron energy dependence of the experimental LRA parameters was also investigated for $s$ and $p$ shells of Ar and Xe atoms.

Recently, the LRA was observed for Ar $2 p$ shells by Heinasmaki et al. [19] using two electrostatic analyzers at the MAX II synchrotron. Their results agree well with our experimental data. Trinter et al. [23] have found LRA for the angular distribution of the ejected photoelectrons and $\mathrm{C}$ $\mathrm{K}$-Auger electrons using left- and right-circularly polarized light and a carbon monoxide molecular target.

The exclusion of the possible instrumental sources that could produce the left-right asymmetry and the experimental results show that the observed left-right asymmetry may be a real physical process. Currently, there is no physical explanation for a nonzero asymmetry parameter which violates space inversion symmetry in the photon-atom interaction. Theoretical models and further experiments are required to understand the origin of the observed left-right asymmetry.

\section{ACKNOWLEDGMENTS}

We would like to give special thanks to Professor S. Aksela and his group in Oulu, Finland, for participating in the development of the experimental setup and scientific cooperation in the data collection at the MAX II synchrotron in Lund, Sweden. We thank the members of the Atomic Physics Division of Atomki for useful discussions. The assistance of the staff of DORIS III at HASYLAB is gratefully acknowledged. This work was supported by the Hungarian Scientific Research Foundation (Grant No. OTKA K104409) and the TÁMOP-4.2.2.A-11/1/KONV-2012-0060 project (cofinanced by EU and the European Social Fund).

\section{APPENDIX}

Here we discuss possible sources of the left-right asymmetry which might be due to experimental or instrumental inaccuracies.

\section{Mechanical inaccuracy}

The shapes of the cylinder and the sphere of the analyzer may differ from the ideal form, being slightly eccentric. According to the precise check of the shape, the mechanical inaccuracy is less than $10 \mu \mathrm{m}$ over the entire circumference of the cylinder and of the sphere for both analyzers (ESA-22L and ESA-22G [3,11]). It may cause less than $5 \times 10^{-5}$ uncertainty in the left-right asymmetry parameter. However, these eccentricities cause the same intensity distortions for the Auger electrons and photoelectrons; therefore, the normalization of the photoelectron intensity to an Auger electron intensity eliminates the influence of the mechanical uncertainty to the left-right asymmetry parameter.

\section{Angular inaccuracy}

An angular difference may exist between the real photon beam propagation direction and the beam axis defined by the spectrometer slit system. This angular misalignment is less than $0.28^{\circ}$ in the present experiment and $0.19^{\circ}$ in the previous ones [3]. The values of the left-right asymmetry parameter caused by these misalignments are $5.9 \times 10^{-7}$ and $3.2 \times 10^{-7}$, respectively. A similar effect can be produced by the azimuthal rotation of the holder of the channeltrons. The possible largest rotation angle relative to the proper position is $0.05^{\circ}$ for both analyzers (ESA-22G and ESA-22L), resulting in not more than $4.9 \times 10^{-8}$ left-right asymmetry.

\section{Off-axis alignment of the spectrometer}

When the axis of the spectrometer does not cross the axis of the photon beam (parallel shift), it may cause an acceptance angle difference between the two spectrometer halves. A $0.5-\mathrm{mm}$ deviation between the two axes may produce about $\Delta \psi=0.05^{\circ}$ and $\Delta \chi=0.02^{\circ}$ differences in the acceptance angles for both analyzers. The value of the left-right asymmetry parameter caused by these differences is about 0.022 . However, our standard normalization procedure (i.e., the intensity of the photoelectrons is normalized to the intensity of the Auger electrons) eliminates the effects of these inaccuracies because the solid angle is the same for Auger electrons and photoelectrons.

\section{Magnetic field}

Both spectrometers (ESA-22L and ESA-22G) are shielded by three layers of $\mu$-metal against the Earth's magnetic field. The value of the residual magnetic field is less than $500 \mathrm{nT}$ in the scattering plane and in the analyzer. Nevertheless, this weak magnetic field rotates slightly the trajectories and therefore may modify the original angular distributions of the electrons. The rotation angles of the electron trajectories are about $0.0053^{\circ}$ and $0.0037^{\circ}$ for 40 - and $80-\mathrm{eV}$ pass energies, respectively. If the electron momentum vector is perpendicular to the magnetic induction vector during the flight of the electrons, the values of the corresponding left-right asymmetry parameter are only $A_{L R}=4 \times 10^{-9}$ and $A_{L R}=2.8 \times 10^{-9}$, respectively. This is even lower than the value determined for the angular uncertainty discussed above.

\section{Contamination of the electrodes}

Dirty surfaces of the analyzer electrodes may produce local charging, which modifies the trajectory and the angular distribution of emitted electrons. This effect increases with decreasing pass energy. The pass energy dependence of the photoelectron angular distribution was checked experimentally at beam line I411 of the MAX II synchrotron using the ESA-22L electron spectrometer [12]. The Ar $2 p$ photoelectron line was measured at $440-\mathrm{eV}$ photon energy with different deceleration ratios $\left(E_{\text {kin }} / E_{\text {pass }}\right)$ which varied between 1 and 9 (20-190-eV pass energy). The values of the left-right asymmetry parameters were constant within $20 \%$ in this wide-pass energy range. This investigation demonstrates the cleanness of the electrode surfaces and excludes any contribution of charging effect to the left-right asymmetry.

\section{Exit slit of the monochromator}

Changing the exit slit size of the monochromator may modify the dimension and the intensity distribution of the photon beam in the target region. This may produce a 
"misalignment" effect similar to the geometrical misalignments discussed above. These investigations were carried out at photon energy where the photon flux had maximum value. The detected count rates of the channeltrons were also maximum in these measurements relative to the "normal" measurements. The count rates of every channeltron varied within a factor of 100 between the minimum and maximum values of the monochromator exit slit size. It was found that the value of the left-right asymmetry parameter does not depend on the slit size of the monochromator within $0.4 \%$ and $2.5 \%$ error in the present and earlier experiments, respectively. This independence also indicates that the signal processing and the speed of the counters were sufficiently fast and dead time effects were negligible.
[1] J. W. Cooper, Phys. Rev. A 42, 6942 (1990).

[2] A. Derevianko and W. R. Johnson, At. Data Nucl. Data Tables 73, 153 (1999).

[3] S. Ricz, T. Ricsóka, Á. Kövér, D. Varga, M. Huttula, S. Urpelainen, H. Aksela, and S. Aksela, New J. Phys. 9, 274 (2007).

[4] G. G. Paulus, F. Grasbon, H. Walther, P. Villoresi, M. Nisoli, S. Stagira, E. Priori, and S. De Silvestri, Nature (London) 414, 182 (2001).

[5] R. Reininger and V. Saile, Nucl. Instrum. Methods Phys. Res., Sect. A 288, 343 (1990).

[6] A. R. B. de Castro and R. Reininger, Rev. Sci. Instrum. 63, 1317 (1992).

[7] T. Möller, Synchrotron Radiat. News 6, 16 (1993).

[8] C. U. S. Larsson, A. Beutler, O. Björneholm, F. Federmann, U. Hahn, A. Rieck, S. Verbin, and T. Möller, Nucl. Instrum. Methods Phys. Res., Sect. A 337, 603 (1994).

[9] M. Bässler, J. O. Forsell, O. Björneholm, R. Feifel, M. Jurvansuu, S. Aksela, S. Sundin, S. L. Sorensen, R. Nyholm, A. Ausmees, and S. Svensson, J. Electron Spectrosc. Relat. Phenom. 101-103, 953 (1999).

[10] M. Bässler, A. Ausmees, M. Jurvansuu, R. Feifel, J. O. Forsell, P. de Tarso Fonseca, A. Kivimäki, S. Sundin, S. L. Sorensen, R. Nyholm, O. Björneholm, S. Aksela, and S. Svensson, Nucl. Instrum. Methods Phys. Res., Sect. A 469, 382 (2001).

[11] T. Ricsóka, S. Ricz, Á. Kövér, S. Schippers, K. Holste, A. Borovik, Jr., D. Varga, and A. Müller, J. Phys.: Conf. Ser. 194, 012003 (2009).
[12] S. Ricz, Á. Kövér, M. Jurvansuu, D. Varga, J. Molnár, and S. Aksela, Phys. Rev. A 65, 042707 (2002).

[13] B. Cleff and W. Mehlhorn, J. Phys. B: At. Mol. Phys. 7, 593 (1974).

[14] E. G. Berezhko and N. M. Kabachnik, J. Phys. B: At. Mol. Phys. 10, 2467 (1977).

[15] W. Mehlhorn, Phys. Lett. A 26, 166 (1968).

[16] E. G. Berezhko, N. M. Kabachnik, and V. S. Rostovsky, J. Phys. B: At. Mol. Phys. 11, 1749 (1978).

[17] L. Sarkadi, T. Vajnai, J. Pálinkás, Á. Kövér, J. Végh, and T. Mukoyama, J. Phys. B: At. Mol. Phys. 23, 3643 (1990).

[18] O. Hemmers, G. Fisher, P. Glans, D. L. Hansen, H. Wang, S. B. Whitfield, R. Wehlitz, J. C. Levin, I. A. Sellin, R. C. C. Perera, E. W. B. Dias, H. S. Chakraborty, P. C. Deshmukh, S. T. Manson, and D. W. Lindle, J. Phys. B: At. Mol. Phys. 30, L727 (1997).

[19] S. Heinasmaki, S. Urpelainen, and M. Huttula, Phys. Scr. 87, 065302 (2013).

[20] S. Ricz, J. Nikkinen, R. Sankari, T. Ricsóka, Á. Kövér, D. Varga, S. Fritzsche, H. Aksela, and S. Aksela, Phys. Rev. A 72, 014701 (2005).

[21] T. W. Gorczyca and F. Robicheaux, Phys. Rev. A 60, 1216 (1999).

[22] S. Ricz, R. Sankari, Á. Kövér, M. Jurvansuu, D. Varga, J. Nikkinen, T. Ricsóka, H. Aksela, and S. Aksela, Phys. Rev. A 67, 012712 (2003).

[23] F. Trinter, L. Ph. H. Schmidt, T. Jahnke, M. S. Schäffler, O. Jagutzki, A. Czasch, J. Lower, T. A. Isaev, R. Berger, A. L. Landers, Th. Weber, R. Dörner, and H. Schmidt-Böcking, Mol. Phys. 110, 1863 (2012). 